\title{
TRPV5 and TRPV6 Channels in Human Myeloid Leukemia Cells
}

\author{
S. B. Semenova, I. O. Vassilieva, and Y. A. Negulyaev \\ Institute of Cytology, Russian Academy of Sciences, St. Petersburg, Russia; \\ e-mail:svsem@mail.cytspb.rssi.ru
}

DOI: $10.1134 / \mathrm{S} 1990747809030441$

Transmembrane channels in blood cells are regulated by receptors, intracellular calcium stores and by different messengers involved in cell signaling. Although the calcium currents in blood cells are well studied, the molecular identity of ionic channels in these cells have not yet been determined. In recent years, a considerable progress was made in molecular and functional characterization of TRP (transient receptor potential) channels. The TRP members display a specific expression pattern, different selectivity, gating, and mode of activating and apparently provide the integration of the different signal pathways in the cells. Although the mRNAs for several types of the TRP family proteins have been found in cells of hematopoietic lineage, functional properties and the role of these plasmalemmal channels in $\mathrm{Ca}^{2+}$ signaling in blood cells remains uncertain. Our data present the first identification of the transient receptor potential of vaniloid type 5 (TRPV5) and 6 (TRPV6) channels in K562 cells. Using RT-PCR analysis, we have shown that human leukemia K562 cells endogenously coexpress the TRPV5 and TRPV6 mRNAs. Moreover, using antiTRPV5 and anti-TRPV6 antibodies we demonstrated that TRPV5 and TRPV6 channel proteins are present both in total lysates and in crude membrane preparations from leukemia cells. Recently in plasma membrane of K562 cells we discovered the endogenous 30-pS cation channels in divalent ion-free solution (DVF). The inward rectification, voltage-dependent gating, and sensitivity to intracellular divalent ions displayed the similarity of these channels with calciumselective channels. It is known that from 29 members of the TRPs superfamily, all of them cation channels, only TRPV5 and TRPV6 exhibit properties of highly selec- tive $\mathrm{Ca}^{2+}$ channels. Here, in outside-out mode we showed that extracellular $\mathrm{Ca}^{2+}$ blocks these channels with $\mathrm{IC}_{50}=20 \mu \mathrm{M}$. Differences in the affinity to extracellular $\mathrm{Ca}^{2+}$ between recombinantly expressed TRPV5 or TRPV6 channels and of the endogenous 30-pS channels in K562 cells indicate that endogenous channels have a specific pore property, which may be a result of heteromultimeric assembly of TRPV5 and TRPV6 proteins. Recently, in expression systems, it has been shown that TRPV5 and TRPV6 can generate a pleiotropic set of functional heterotetrameric channels with different $\mathrm{Ca}^{2+}$ transport kinetics. We have also found that RR, a nonspecific blocker of TRPV5 and TRPV6, blocks the endogenous 30-pS channel activity with $\mathrm{IC}_{50}=6.5 \mu \mathrm{M}$ in $\mathrm{K} 562$ cells. This value is slightly lower than the $\mathrm{IC}_{50}$ reported for the RR block of the recombinant TRPV6 channels and higher than that of the recombinant TRPV5 channels. Thus, taken together, our data indicate that TRPV5 and TRPV6 proteins are endogenously coexpressed and presumably form functional heterotetrameric TRPV5/V6 channels in K562 cells, which contain more TRPV6 subunits than those of TRPV 5 type. We speculate that variations in subunit composition of TRPV5 and TRPV 6 channels could provide a mechanism for a fine tuning of $\mathrm{Ca}^{2+}$ transport kinetics and $\mathrm{Ca}^{2+}$-dependent functions, such as proliferation and differentiation, in human myeloid leukemia cells.

The work was supported by the Russian Foundation for Basic Research (project nos. 07-04-00679 and 0804-00659) and Molecular and Cell Biology Program of the Russian Academy of Sciences. 